\title{
Dynamic Jiles-Atherton Model for Determining the Magnetic Power Loss at High Frequency in Permanent Magnet Machines
}

\author{
Ruoyang $\mathrm{Du}^{1}$, Student Member, IEEE, and Paul Robertson ${ }^{1}$ \\ ${ }^{1}$ Electrical Engineering Division, University of Cambridge, Cambridge, CB3 0FA,UK
}

\begin{abstract}
This paper describes a mathematical model for the dynamic magnetic power losses in the laminated steel stator of high frequency permanent magnet machines, such as Brushless DC (BLDC) Motors. The model presented is based on a utilization of the dynamic Jiles-Atherton model. Accurate dynamic BH curve fitting and magnetic power loss derivations have been achieved, where the calculated magnetic losses have shown around $95 \%$ accuracy from $5 \mathrm{~Hz}$ to $2000 \mathrm{~Hz}$, over a flux density range of $1.0 \mathrm{~T}$ to $1.6 \mathrm{~T}$. This approach has been applied to estimate the magnetic power loss of a small scale, high frequency $(>10,000 \mathrm{rpm}) \mathrm{BLDC}$ motor, with calculated and measured losses being in close agreement.
\end{abstract}

Index Terms - Finite element methods (FEM), Magnetic hysteresis, Magnetic losses, Magnetization, Permanent magnet machines, Permanent magnet motors, Permanent magnet generators.

\section{INTRODUCTION}

$\mathrm{T}$ HE magnetic power losses in ferromagnetic materials have been continuously studied since the first formulation of power loss in ferromagnetic materials was derived by Steinmetz [1]. According to more recent research [2],[3], the magnetic losses are normally decomposed into the sum of a frequency independent hysteresis contribution, $\mathrm{P}_{\text {hysteresis }}$, and a frequency dependent dynamic contribution, $\mathrm{P}_{\text {dynamic }}$.

On one hand, considering magnetic domain wall theory, the hysteresis contribution $\mathrm{P}_{\text {hysteresis }}$ corresponds to domain wall displacement against the pinning effect [4]. On the other hand, in terms of the physical origin of the dynamic contribution $\mathrm{P}_{\text {dynamic }}$, it was considered as the sum of the classic eddy current loss [5], [6], and a supplementary term of excess or anomalous loss[7],[8], [9]:

$$
\begin{aligned}
\mathbf{P}_{v}=\mathbf{P}_{\text {hysteresis }} & +\mathbf{P}_{\text {eddy }}+\mathbf{P}_{\text {anomalous }} \\
& =\mathbf{k}_{\mathbf{h}} \mathbf{f}_{\mathbf{m}} \mathbf{B}^{\mathbf{n}}+\mathbf{k}_{\mathbf{e}} \mathbf{f}_{\mathbf{m}}^{2} \mathbf{B}^{2}+\mathbf{k}_{\mathbf{a}} \mathbf{f}_{\mathbf{m}}^{1.5} \mathbf{B}^{1.5}(1)
\end{aligned}
$$

Where $\mathrm{P}_{v}$ is the total magnetic loss per unit volume, $\mathrm{k}_{\mathrm{h}}, \mathrm{k}_{\mathrm{e}}$, and $\mathrm{k}_{\mathrm{a}}$ are the coefficients of the hysteresis loss, eddy current loss, and anomalous loss, $f_{m}$ is the frequency of the magnetic field, and B is the induction flux density [10].

However, this formula has been proven inappropriate for non-oriented laminations when the magnetic field frequency $f_{m}$ is higher than $400 \mathrm{~Hz}$ [11]. It is surmised that the discontinuous character of the magnetization process at the microscopic level is a nonlinear and complex function of magnetization and frequency [3], [9], [12], and that the hysteresis loss is influenced by the dynamic loss at high frequency [3]; which means the interactions of the three terms in (1) need to be taken into account for high frequency calculations.

In this paper, we introduce a transient mathematical model which can automatically take the interactions of the three terms in (1) into account. This model is able to calculate the $\mathrm{BH}$ curves and magnetic power losses in laminated steel accurately, over a wide range of frequencies between $5 \mathrm{~Hz}$ and $2,000 \mathrm{~Hz}$ and a magnetic flux density from 1.0T to 1.6T i.e. the conditions typically found in a modern BLDC motor. The key elements of this model are the Jiles-Atherton Model [4] and its extension to conducting magnetic materials [13], which will be called the 'dynamic Jiles-Atherton model' in this paper. Several necessary modifications have been made to the original dynamic Jiles-Atherton model in order to make it work correctly for laminations in high frequency permanent magnet synchronous machines (PMSMs), such as BLDC motors.

Since the working frequencies of modern BLDC motors are normally from hundreds to thousands $\mathrm{Hz}$, the conventional power loss formulae for $50 \mathrm{~Hz}$ are no longer appropriate for this kind of electrical machine [11].Our proposed dynamic Jiles-Atherton model offers an accurate mathematical approach for modeling the magnetic power loss in this kind of machine, over a wide range of magnetic working frequencies and flux densities. Only two BH curves are needed for any given $B_{\max }$ to simulate the dynamic properties of the selected laminated steel.

\section{MATHEMATICAL MODELS}

\section{A. Static Jiles-Atherton Model}

Jiles and Atherton [4] proposed a mathematical model of the hysteresis mechanism in ferromagnetic materials, based on physical insights into the magnetization process. The five original equations of Jiles-Atherton model are listed below:

$$
\mathbf{M}_{\mathrm{an}}\left(\mathbf{H}_{\mathrm{e}}\right)=\mathbf{M}_{\mathbf{s}}\left(\operatorname{coth}\left(\mathbf{H}_{\mathrm{e}} / \boldsymbol{a}\right)-\left(\boldsymbol{a} / \mathbf{H}_{\mathrm{e}}\right)\right)
$$

Where $M_{a n}$ is the anhysteretic magnetization, $H_{e}$ is the effective field, $M_{s}$ is the saturation magnetization, and $a$ is a parameter with dimensions of magnetic field, which characterizes the shape of the anhysteretic magnetization.

$$
H_{e}=H+\alpha M
$$

Where $H$ is the applied field, $M$ is the bulk magnetization, and $\alpha$ is a mean field parameter representing interdomain coupling.

$$
\mathbf{M}=\mathbf{M}_{\text {rev }}+\mathbf{M}_{\mathrm{irr}}
$$


Where $M_{\text {rev }}$ is the reversible magnetization, and $M_{\text {irr }}$ is the irreversible magnetization.

$$
\mathbf{M}_{\text {rev }}=\mathbf{c}\left(\mathbf{M}_{\mathrm{an}}-\mathbf{M}_{\mathrm{irr}}\right)
$$

Where $\mathrm{c}$ is the coefficient of proportionality which can be determined experimentally by the ratio of the initial differential susceptibilities of the normal and anhysteretic magnetization curves [4],[14].

$$
\begin{aligned}
\mu_{0} \int M_{a n} d_{H_{e}=} & \mu_{0} \int M d H_{e} \\
& +\mu_{0} k \delta(1-c) \int\left(\frac{d M_{i r r}}{d H_{e}}\right) d H_{e}
\end{aligned}
$$

Where the coefficient $\mathrm{k}$ is the pining parameter which determines the amount of energy dissipated, and $\delta$ is a directional parameter which ensures that energy is always lost through dissipation; which means $\delta=+1$ when $\mathrm{dH} / \mathrm{dt} \geq 0$ and $\delta=-1$ when $\mathrm{dH} / \mathrm{dt}<0$.

According to the explanation above, (2), (3), (4), (5) \& (6) are the five original equations of the Jiles-Atherton model. Jiles and Atherton initially assumed that $\mathrm{c}=0$ and $\mathrm{M}_{\text {rev }}=0$ in (6) in the first edition of the Jiles-Atherton model [4]. This assumption makes $\mathrm{M}=\mathrm{M}_{\mathrm{irr}}$, and leads to the differential equation for hysteresis:

$$
\frac{d M}{d H}=\frac{1}{(1+c)} \frac{M_{a n}-M}{k \delta-\alpha\left(M_{a n}-M\right)}+\frac{c}{(1+c)} \frac{d M_{a n}}{d H}
$$

Where the parameter $\mathrm{c}$ in (7) comes only from (5).

However, according to domain wall theory, domain wall bending will normally occur in ferromagnetic materials [4], which means that the assumption of $M_{\text {rev }}=0$ is not necessarily valid. Additionally, the value of $\mathrm{c}$ is significant in all the measured materials in a later publication by Jiles[14], which is normally 0.14 to 0.55 within the maximum bounds from 0 to 1 . Under these circumstances, Jiles abandoned the assumptions that $\mathrm{c}=0$ and $\mathrm{M}_{\text {rev }}=0$ when he tried to extend the static Jiles-Atherton model into a dynamic model [8], in other words, the static Jiles-Atherton model is not consistent with the dynamic Jiles-Atherton model [13].

Therefore, in order to avoid this inconsistency, we rederived the static Jiles-Atherton model based on the five original equations mentioned above without the assumption that $\mathrm{c}=0$ and $\mathrm{M}_{\mathrm{rev}}=0$. Thus, the re-derived differential equation for magnetic hysteresis is:

$$
\frac{\mathbf{d M}}{\mathbf{d H}}=\frac{\mathbf{M}_{\mathrm{an}}-\mathbf{M}}{\mathbf{k} \boldsymbol{\delta}-\boldsymbol{\alpha}\left(\mathbf{M}_{\mathrm{an}}-\mathbf{M}\right)}+\frac{\mathbf{c k} \boldsymbol{k} \boldsymbol{\mathbf { k }}-\boldsymbol{\alpha}\left(\mathbf{M}_{\mathrm{an}}-\mathbf{M}\right)}{\mathbf{d H}}
$$

And by substituting (3) into (2):

$$
\mathbf{M}_{\mathrm{an}}=\mathbf{M}_{\mathbf{s}}\left(\operatorname{coth}\left(\frac{\mathbf{H}+\boldsymbol{\alpha M}}{a}\right)-\left(\frac{a}{\mathbf{H}+\boldsymbol{\alpha M}}\right)\right)
$$

The five original equations of the Jiles-Atherton model have been simplified into two equations; which are (8) and (9). This re-derived static Jiles-Atherton model is consistent with the dynamic Jiles-Atherton model [13] in our simulations.

As seen in (8) and (9), there are in total five parameters $\alpha, a, c, k, M_{s}$ in the re-derived Jiles Atherton model. According to Jiles [13], $\mathrm{B}=\mu_{0}(\mathrm{M}+\mathrm{H}) \cong \mu_{0} \mathrm{M}$ in laminations because $\mathrm{H} \ll \mathrm{M}$ in soft magnetic materials, so $\mathrm{M}_{\mathrm{s}} \cong \mathrm{B}_{\mathrm{s}} / \mu_{0}$ will apply in laminations, where $\mathrm{B}_{\mathrm{s}}$ is the saturation flux density given by the manufacturer. Therefore, there are actually four parameters $\alpha, \mathrm{a}, \mathrm{c} \& k$ which need to be determined for the re-derived Jiles-Atherton model. The numerical method for the parameter identification can be found in Jiles' paper [14].

In order to simplify the experimental system and increase the BH curve fitting accuracy of the parameter identification process, several evolutionary computing methods have been previously used, based on the iterative trial and error basis using measured hysteresis loops [15], [16], [17], [18], [19], [20]. We selected particle swarm optimization (PSO) [21], [22] as our approach because it can achieve better accuracy based on simpler tuning and less iteration time compared with other approaches [20]. The tuning of the PSO method in this application is shown in the Appendix A.

\section{B. Dynamic Jiles-Atherton Model}

Jiles has already attempted to illustrate the frequency dependence of hysteresis curves in conducting magnetic materials [13]. The classical eddy current instantaneous power loss per unit volume is proportional to the square of the rate of change of magnetization as discussed by Chikazumi [5], [13]. This gives:

$$
\frac{d W_{\text {eddy }}}{d t}=\frac{d^{2}}{2 \rho \beta} \cdot\left(\frac{d B}{d t}\right)^{2}=\frac{\mu_{0}^{2} d^{2}}{2 \rho \beta} \cdot\left(\frac{d M}{d t}\right)^{2}
$$

Where $\mathrm{W}_{\text {eddy }}$ is the dissipation due to eddy current loss, $\rho$ is the resistivity in $\Omega \mathrm{m}, \mathrm{d}$ is the cross-sectional dimension in meters; which is thickness for laminations or diameter for cylinders and spheres. $\beta$ is a geometrical factor which varies from $\beta=6$ in laminations, $\beta=16$ in cylinders and $\beta=20$ in spheres[13].

The expression of the anomalous instantaneous power loss with respect to magnetic induction derivative $d B / d t$ has been developed and justified by Fiorillo and Novikov [12]. The anomalous instantaneous power loss per unit volume can be expressed as:

$$
\begin{aligned}
\frac{d W_{\text {anomalous }}}{d t}=( & \left.\frac{G d w H_{0}}{\rho}\right)^{1 / 2} \cdot\left(\frac{d B}{d t}\right)^{3 / 2} \\
& =\left(\frac{G d w H_{0}}{\rho}\right)^{1 / 2} \cdot\left(\frac{\mu_{0} d M}{d t}\right)^{3 / 2}
\end{aligned}
$$

Where $\mathrm{W}_{\text {anomalous }}$ is the dissipation due to anomalous loss, $\mathrm{G}$ is a dimensionless constant of value 0.1356 [12], $\mathrm{w}$ and $\mathrm{d}$ are the width and thickness of the laminations. $\mathrm{H}_{0}$ is a parameter representing the fluctuating internal potential experienced by domain walls [9].

However, the original dynamic Jiles-Atherton model has not been commonly used in practice due to its relatively low accuracy. The major problem with the original dynamic JilesAtherton model is that the pre-determined parameter $\mathrm{H}_{0}$ is not actually constant in this transient mathematical model, but 
should be determined based on each individual case; which is solved using the PSO method in our work.

For the excess loss in (11), Jiles calculated the crosssectional area $\mathrm{S}$ as $(\mathrm{w} \times \mathrm{d})$, which is the cross-sectional area of one layer of the laminations. Actually, one layer of lamination is not normally used in practical applications. Additionally, Jiles has missed out the absolute value sign for $\mathrm{dB} / \mathrm{dt}$ [12], which will cause unphysical behavior when the value of $\mathrm{dB} / \mathrm{dt}$ becomes negative. Therefore, the anomalous loss (11) should be modified to:

$$
\begin{aligned}
\frac{\mathrm{dW}_{\text {anomalous }}}{\mathrm{dt}}= & \left(\frac{\mathrm{GSH}_{0}}{\rho}\right)^{1 / 2} \cdot\left|\frac{\mathrm{dB}}{\mathrm{dt}}\right|^{3 / 2} \\
& =\left(\frac{\mathrm{GSH}_{0}}{\rho}\right)^{1 / 2} \cdot\left|\frac{\mu_{0} \mathrm{dM}}{\mathrm{dt}}\right|^{3 / 2}
\end{aligned}
$$

Where $S$ is the cross-sectional area of the laminated steel stack.

Taking both classic eddy current loss and anomalous loss into consideration, the energy balance equation of the hysteresis (6) can be extended to [13]:

$$
\begin{aligned}
\mu_{0} \int M_{\mathrm{an}}(\mathrm{H}) \mathrm{dH}_{\mathrm{e}} & \\
& =\mu_{0} \int \mathrm{M}(\mathrm{H}) \mathrm{dH}_{\mathrm{e}}+\mu_{0} \mathrm{k} \delta(1 \\
& -\mathrm{c}) \int\left(\frac{\mathrm{dM}_{\mathrm{irr}}}{\mathrm{dH}}\right) \mathrm{d} \mathrm{H}_{\mathrm{e}} \\
& +\int \frac{\mu_{0}^{2} \mathrm{~d}^{2}}{2 \rho \beta}\left(\frac{\mathrm{dM}}{\mathrm{dt}}\right)^{2} \mathrm{dt} \\
& +\int\left(\frac{\mathrm{GSH}_{0}}{\rho}\right)^{\frac{1}{2}}\left|\frac{\mu_{0} d M^{\frac{3}{2}}}{\mathrm{dt}}\right|^{\frac{3}{2}} \mathrm{dt}
\end{aligned}
$$

In accordance with Jiles' paper [13], the left-hand side corresponds to the energy of the lossless anhysteretic process $\mathbf{W}_{\mathrm{an}}$, the first term on the right-hand side is the contribution to the magnetostatic energy $\mathbf{W}_{\mathrm{M}}$, the second, third, and fourth terms on the right-hand side are the dissipation due to domain wall pining loss $\mathbf{W}_{\text {hysteresis }}$, the dissipation due to eddy current loss $\mathbf{W}_{\text {eddy }}$, and the dissipation due to anomalous loss $\mathbf{W}_{\text {anomalous }}$, respectively. If there is no dissipation, the magnetization must follow the anhysteretic curve. This equation can be manipulated to:

$$
\begin{aligned}
M_{\mathrm{an}}(\mathrm{H})=\mathrm{M}(\mathrm{H}) & +\mathbf{k} \delta(1-\mathbf{c})\left(\frac{\mathrm{dM}_{\mathrm{irr}}}{\mathrm{dH}_{\mathrm{e}}}\right)+\frac{\mu_{0} \mathrm{~d}^{2}}{2 \rho \beta}\left(\frac{\mathrm{dM}}{\mathrm{dt}}\right)\left(\frac{\mathrm{dM}}{\mathrm{dH}_{\mathrm{e}}}\right) \\
& +\left(\frac{\mathrm{GSH}_{0} \mu_{0}}{\rho}\right)^{\frac{1}{2}}\left|\frac{\mathbf{d M}}{\mathrm{dt}}\right|^{\frac{1}{2}}\left(\frac{\mathbf{d M}}{\mathrm{dH}_{\mathrm{e}}}\right)
\end{aligned}
$$

Equation (14) can be directly solved together with (2), (3), (4) \& (5) using numerical methods.

It can be seen that there is only one additional parameter $\mathrm{H}_{0}$ which needs to be determined in the dynamic model. Therefore, we can measure one additional high frequency $\mathrm{BH}$ curve under the same $B_{\max }$ as the static measurement, and use the PSO method to find it. The details of the tuning of the PSO method are shown in the Appendix B.

\section{ApPlication of MAthematical Models}

\section{A. Application of the Dynamic Jiles-Atherton Model to Magnetic Loss for Alternating Magnetic Fields}

The original Jiles-Atherton model [4] was proposed to model the saturated static hysteresis mechanism in ferromagnetic materials, and the five parameters $\alpha, \mathrm{a}, \mathrm{c}, \mathrm{k} \& \mathrm{M}_{s}$ were set as constants for each kind of material [14]. Actually, even though we use the same material, our experimental results reveal that a different number of layers in the laminated steel stack will result in slightly different BH curves, where the thicker lamination stacks need a lower excitation field to achieve the same $B_{\max }$. This phenomenon may be caused by flux leakage from the toroid stack, the alignment of displaced magnetic domains or material sample variations. These factors make each lamination stack a unique case, which therefore needs to be calculated individually.

Furthermore, the five static parameters are not constants and will gradually change when the maximum magnetic flux density $\mathrm{B}_{\max }$ changes. In order to deal with this issue, several improved static models have been proposed to take into account the influence of $\mathrm{B}_{\max }[15]$, [23], [24], [25]. However, none of these improved models are used in our work as they are not consistent with the dynamic Jiles-Atherton described above.

Instead, we decided to use the method of a parameter array[15] in this work. Given that the saturated magnetic flux density is $B_{\text {sat }}$, we divide the maximum magnetic flux density $\mathrm{B}_{\max }$ into small steps within the range from $0.5 \mathrm{~B}_{\text {sat }}$ to $0.8 \mathrm{~B}_{\text {sat }}$, and perform the parameter optimization separately for every measured magnetic flux step, to obtain a parameter array for the static Jiles-Atherton model. The same array will be used in the dynamic Jiles-Atherton model, and the additional parameter $\mathrm{H}_{0}$ will be determined based on a high frequency dynamic BH curve at the same magnetic flux density step. Therefore, as long as the parameter array is determined, the dynamic Jiles-Atherton model will be able to calculate the dynamic $\mathrm{BH}$ curves at frequencies up to several kilohertz.

\section{B. Application of the Dynamic Jiles-Atherton Model to the Magnetic Loss of a PMSM (BLDC motor)}

According to Guo's review paper, empirical formulae have been derived to model the magnetic properties under a rotating magnetic field in order to calculate the magnetic power loss in electrical machines, but their measurement techniques and modeling approaches are still far from standardization [26]. In this paper, we developed a new approach instead of the conventional time stepped FEM [11], [26], [27] to utilize the proposed dynamic Jiles-Atherton model to estimate the magnetic power loss in a PMSM. More mathematical work needs to be done to make the dynamic Jiles-Atherton model and time stepped FEM compatible with each other. Our proposed new approach is computationally fast (as the flux density distribution within the stator geometry is only calculated once by FEM) and yields accurate results over a wide speed range for the PMSM.

Generally, in commercial PMSMs, the geometry of the 
laminations is designed to concentrate the magnetic flux within the winding areas, and the flux density at other positions is invariably much lower. This characteristic also concentrates the magnetic power losses within the winding areas. An example of the calculated flux density distribution (using FEM) of a commercial BLDC motor can be seen in Fig. 1. Furthermore, the flux density in the winding area is almost purely alternating due to the geometric design, as shown by the plot in Fig. 2. These two factors mean that the magnetic power loss in the PMSM is predominantly due to the alternating flux. Therefore, provided we can neglect the relatively small but unknown power loss difference between alternating and rotational fluxes, then the dynamic JilesAtherton model is applicable to modeling the power loss of PMSMs.

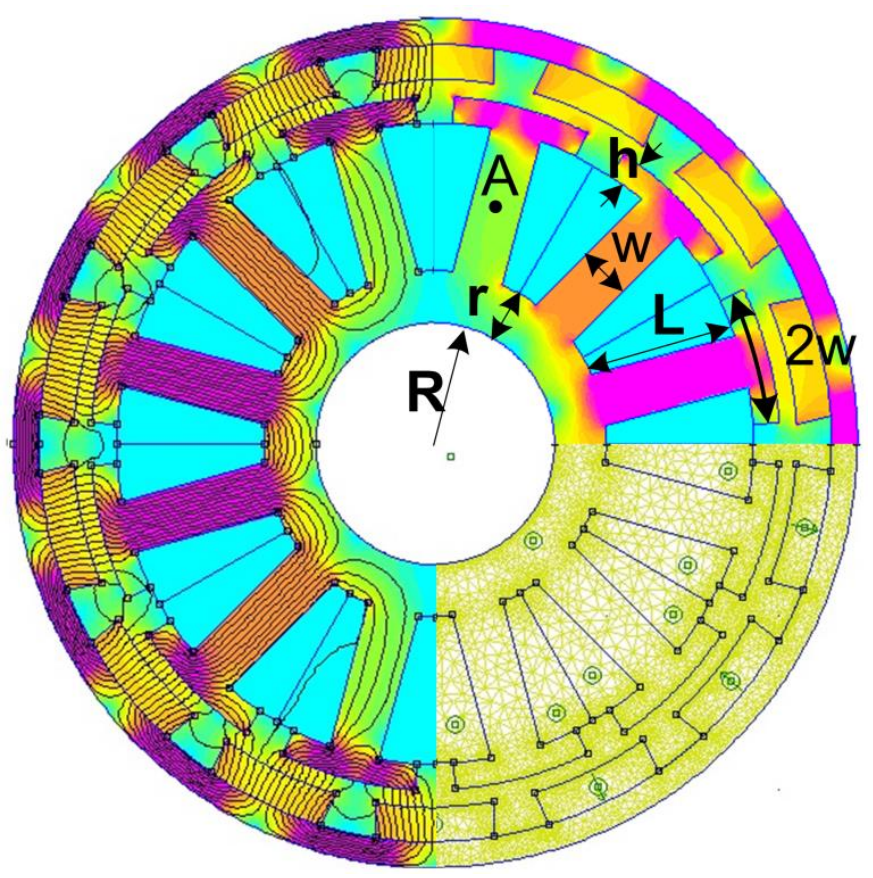

Fig. 1 Section of the Measured Commercial BLDC Motor (Stator Diameter $\varnothing=40 \mathrm{~mm}$ )

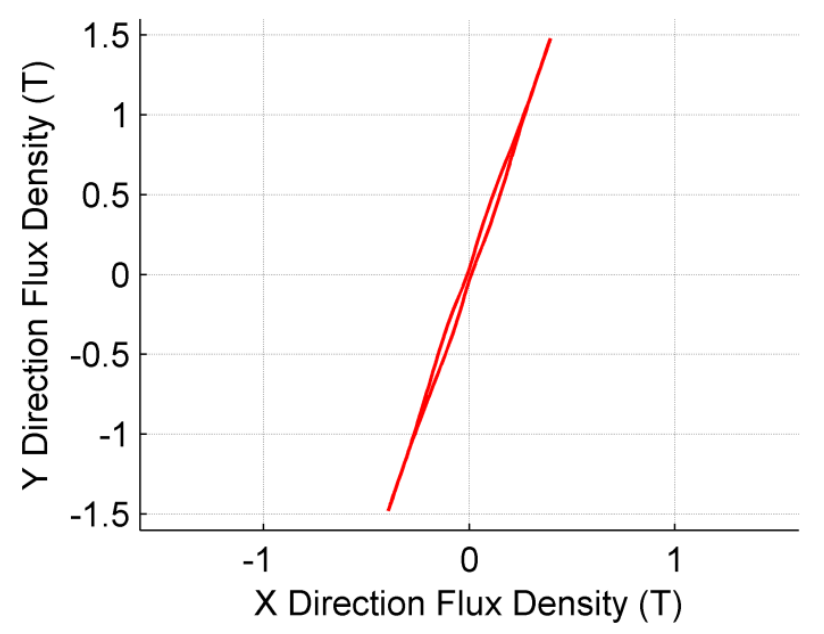

Fig. 2 Stepped Finite Element Method Analysis of the Magnetic Flux Density at Point A

According to our experimental measurements, when the maximum magnetic flux density decreases from $B_{\max }$ to $0.5 \mathrm{~B}_{\max }$, the total magnetic loss will decrease from $\mathrm{P}_{\max }$ to around $0.1 \mathrm{P}_{\max }$. This indicates that the power loss in low flux density elements is negligible compared to that in the high flux density elements. Therefore, we calculated the equivalent volume which encounters the maximum flux density $B_{\max }$, and used it as the functional volume to calculate the total magnetic loss with the dynamic Jiles-Atherton model.

As shown in Fig. 1, the peak flux density will reach $B_{\max }$ in the whole volume of each winding branch when it is lined up with the magnet, so they are $100 \%$ functional. Actually, we might slightly overestimate the power loss in the winding branches because a small proportion of the elements at the edges do not encounter $B_{\max }$. However, the total volume at the edges is much smaller than that of the branches, which makes the difference negligible compared to the total magnetic loss. In the middle circular ring, the magnetic flux of a winding branch will separate into two paths and go into two neighboring winding branches through the ring. Since the flux will tend to go through the outside of the ring, we can assume an outside functional ring, and the thickness of that functional ring should be $0.5 \mathrm{w}$, if we assume that all the flux is concentrated to reach $B_{\max }$. In practice, the flux density between the branches is lower than $0.5 \mathrm{~B}_{\max }$ in the FEM simulation, wherein the loss is negligible compared to the loss in the elements with $B_{\max }$. Therefore, we should subtract this volume from the total functional ring, and this will reduce the volume of the functional ring by about $50 \%$. Hence, assuming the thickness of the lamination is $\mathrm{D}$, the total functional volume can be derived as:

$$
V_{\text {fun }}=\left\{\begin{array}{c}
0.5 \times \pi \\
{\left[(R+r)^{2}-(R+r-0.5 w)^{2}\right]+12 \times L} \\
\times w+12 \times h \times 2 w\} \times D
\end{array}\right.
$$

Then the total magnetic loss can be calculated as:

$$
\boldsymbol{P}=\boldsymbol{A}_{\boldsymbol{B H}} \cdot \boldsymbol{V}_{\boldsymbol{f u n}} \cdot \boldsymbol{f}_{\boldsymbol{m}}
$$

Where $A_{B H}$ is the area within the $\mathrm{BH}$ curve at the magnetic field frequency $f_{m}$.

\section{EXPERIMENTAL RESULTS}

\section{A. Core Losses under Alternating Magnetic Field and Agreement with Modeled Values}

In order to measure the $\mathrm{BH}$ curves of the steel laminations under alternating magnetic fields, we extracted the laminated stator core stacks from commercial motors and incorporated them into the test system shown in Fig. 3. Raw data has been downloaded from the oscilloscope and processed in Matlab to derive the measured BH curves. This is similar to a test system previously described by other researchers [28]. 


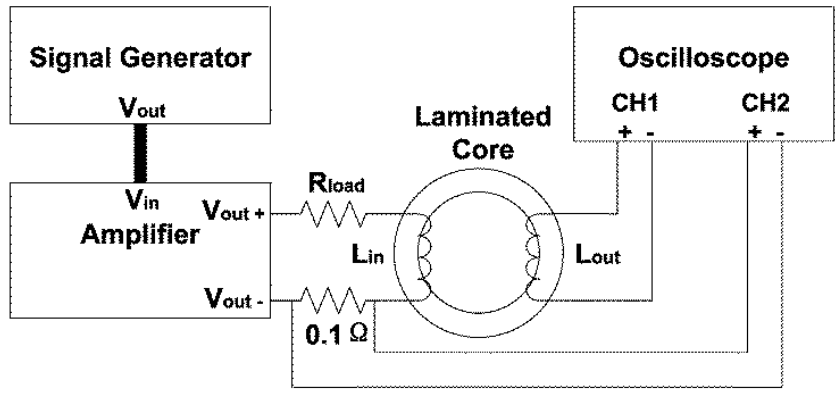

Fig. 3 BH Curve Test System for the Laminated Core

The lowest working frequency of our system is $5 \mathrm{~Hz}$, due to the bandwidth limitation of the power amplifier. We have measured the $\mathrm{BH}$ curves at $5 \mathrm{~Hz}$ and $10 \mathrm{~Hz}$, and found that relative to the $400+\mathrm{Hz} \mathrm{BH}$ curves, the difference between the shapes of the $5 \mathrm{~Hz}$ and $10 \mathrm{~Hz}$ curves is small, and we therefore assume that the $5 \mathrm{~Hz}$ values can adequately represent the static BH curve. Other researchers have found similar results [29], [30], [31]. However, we do see a decline in modeling accuracy at lower frequencies which may be due, at least in part, to this assumption - this is discussed in more detail later.

We measured two laminated stator core stacks with the same section as shown in Fig. 1 but with different thicknesses, which are $10 \mathrm{~mm}$ and $20 \mathrm{~mm}$, and we use the $20 \mathrm{~mm}$ stack here as an example.

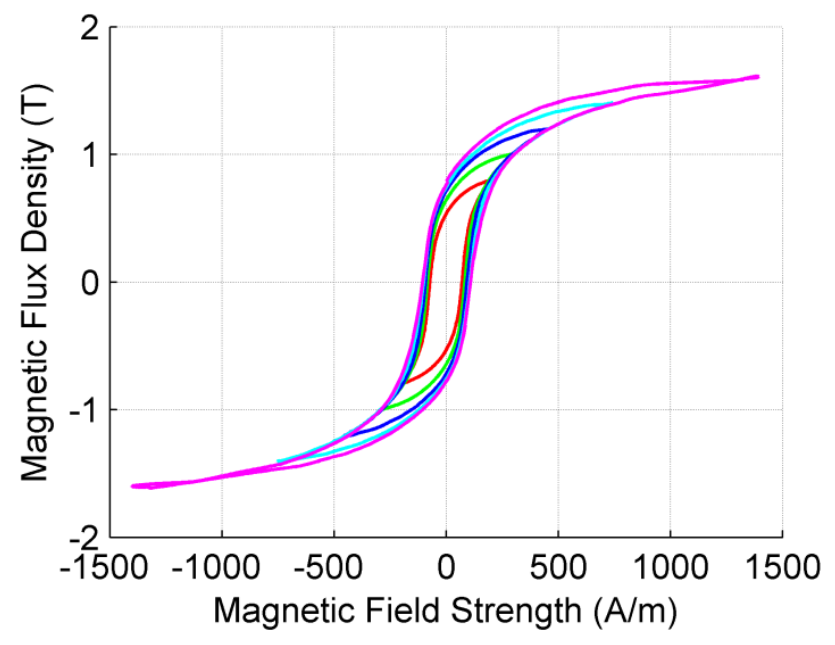

Fig. 4 Measured $5 \mathrm{~Hz}$ BH Curves of 20mm Core

To reduce the experimental noise, our measured results are filtered by the Matlab built-in block encompassing the Savitzky-Golay method [32]. The static Jiles-Atherton parameters are extracted by the PSO method in the Appendix A, with the results shown in Table 1.

Table 1

JILES-ATHERTON PARAMETERS FOR 20 MM NON-
ORIENTED LAMINATION STACK BY PSO
\begin{tabular}{|ccccc}
\hline $\mathrm{B}_{\max }[\mathrm{T}]$ & $\alpha$ & $\mathrm{a}$ & $\mathrm{c}$ & $\mathrm{k}$ \\
\hline 1.0 & $1.222 \mathrm{e}-03$ & $6.934 \mathrm{e}+02$ & $1.796 \mathrm{e}-01$ & $1.002 \mathrm{e}+02$ \\
1.2 & $1.076 \mathrm{e}-03$ & $6.148 \mathrm{e}+02$ & $1.626 \mathrm{e}-01$ & $1.116 \mathrm{e}+02$
\end{tabular}

\begin{tabular}{lllll}
1.4 & $9.624 \mathrm{e}-04$ & $5.571 \mathrm{e}+02$ & $1.480 \mathrm{e}-01$ & $1.242 \mathrm{e}+02$ \\
1.6 & $9.101 \mathrm{e}-04$ & $5.317 \mathrm{e}+02$ & $1.140 \mathrm{e}-01$ & $1.257 \mathrm{e}+02$ \\
\hline
\end{tabular}

Another high frequency dynamic $\mathrm{BH}$ curve is needed to determine the dynamic parameter $\mathrm{H}_{0}$ for each $\mathrm{B}_{\max }$ value through the PSO method described in Appendix B. The total magnetic loss per unit volume can be calculated as

$$
\begin{aligned}
\boldsymbol{P}_{\boldsymbol{v}}=\boldsymbol{A}_{\boldsymbol{B} \boldsymbol{H}} \cdot \boldsymbol{f}_{\boldsymbol{m}} & \left(\mathrm{W} / \mathrm{m}^{3}\right) \\
& =\mathbf{1} \times \mathbf{1 0}^{-\mathbf{9}} \cdot \boldsymbol{A}_{\boldsymbol{B} \boldsymbol{H}} \cdot \boldsymbol{f}_{\boldsymbol{m}}\left(\mathrm{W} / \mathrm{mm}^{3}\right)
\end{aligned}
$$

Where $A_{B H}$ is the area within the $\mathrm{BH}$ curve at the magnetic field frequency $f_{m}$.

The results are shown in Fig. 5 to Fig. 12.

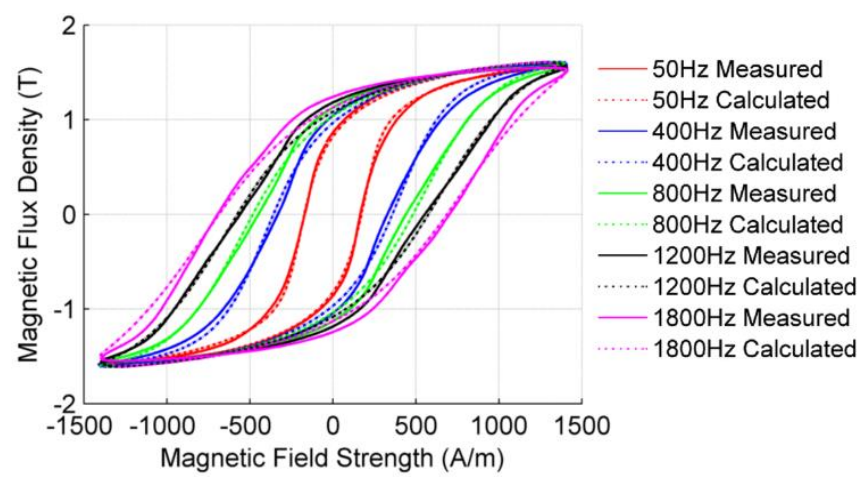

Fig. 5 Measured and Calculated BH Curves of $20 \mathrm{~mm}$ Core $\left(B_{\max }=1.6 T, H_{0}=1.23 \times 10^{-4}\right)$

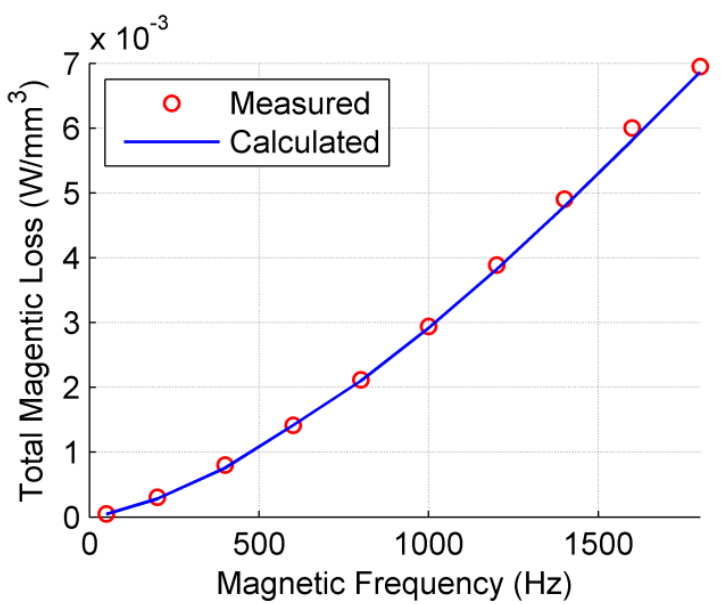

Fig. 6 Dynamic Magnetic Power Loss for $20 \mathrm{~mm}$ Core $\left(B_{\text {max }}=1.6 T, H_{0}=1.23 \times 10^{-4}\right)$

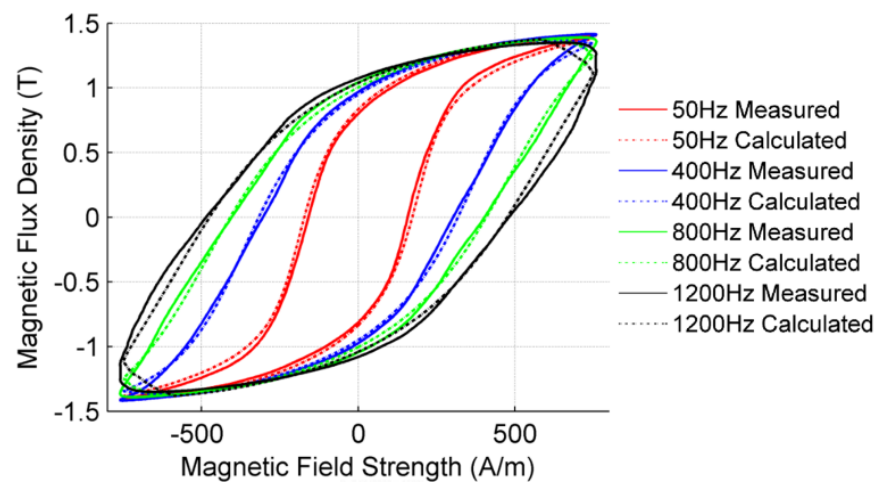


Fig. 7 Measured and Calculated BH Curves of $20 \mathrm{~mm}$ Core $\left(B_{\max }=1.4 T, H_{0}=3.30 \times 10^{-2}\right)$

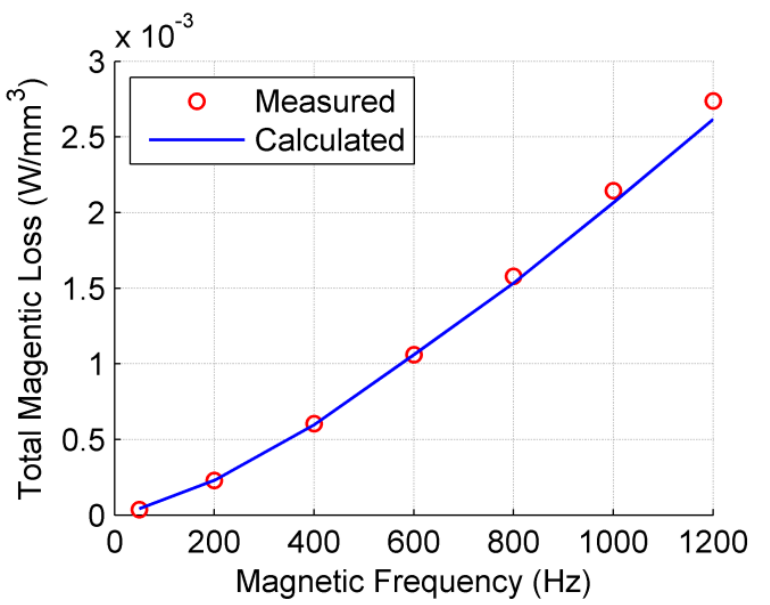

Fig. 8 Dynamic Magnetic Power Loss for $20 \mathrm{~mm}$ Core $\left(B_{\max }=1.4 T, H_{0}=3.30 \times 10^{-2}\right)$

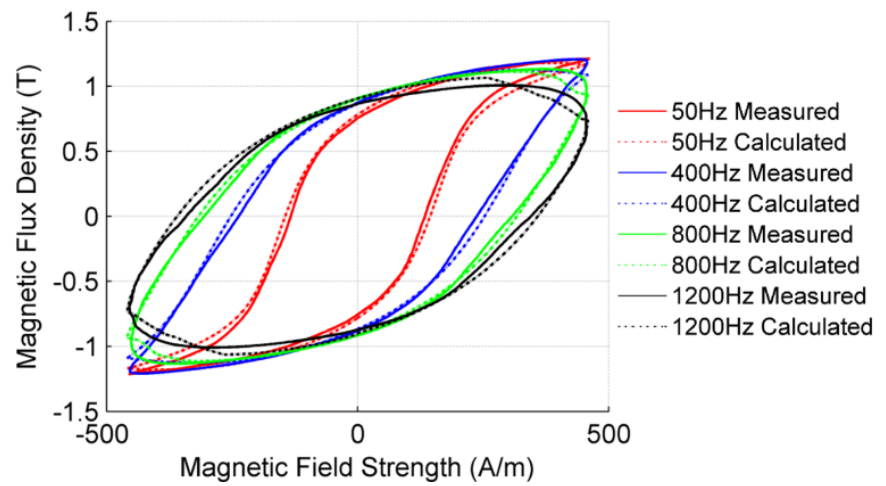

Fig. 9 Measured and Calculated BH Curves of $20 \mathrm{~mm}$ Core $\left(B_{\text {max }}=1.2 \mathrm{~T}, \mathrm{H}_{0}=2.95 \times 10^{-2}\right)$

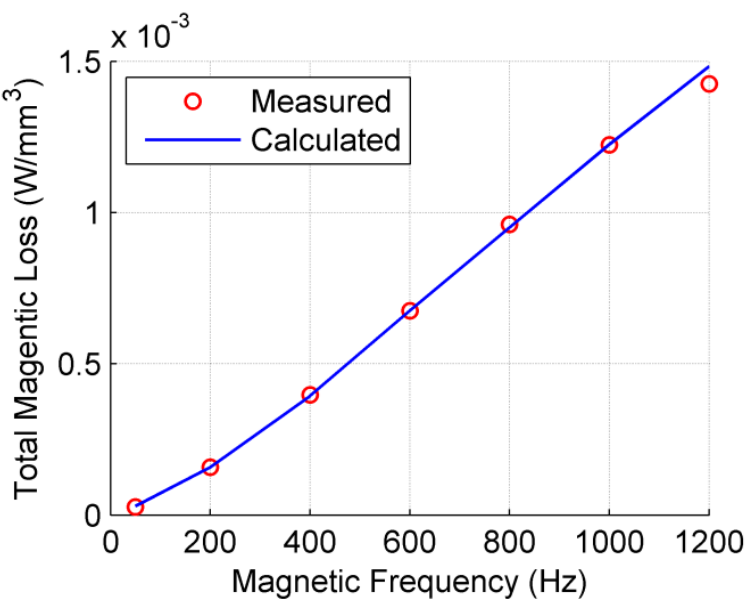

Fig. 10 Dynamic Magnetic Power Loss for $20 \mathrm{~mm}$ Core $\left(B_{\max }=1.2 T, H_{0}=2.95 \times 10^{-2}\right)$

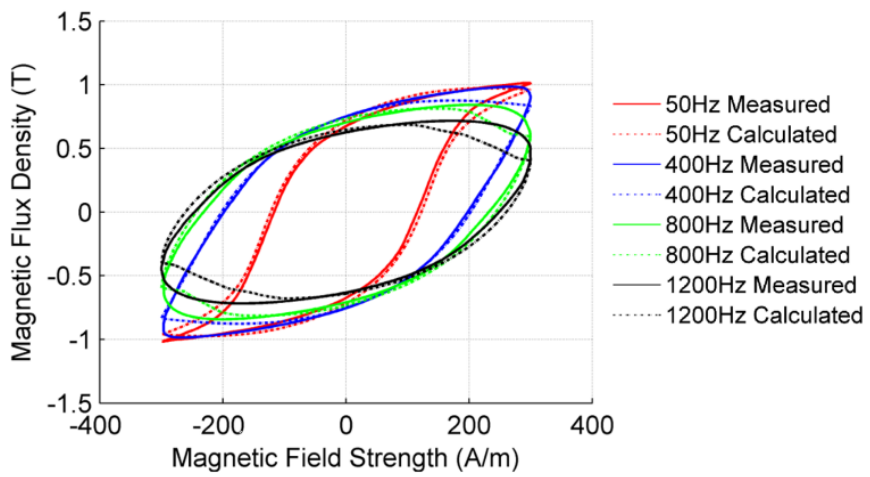

Fig. 11 Measured and Calculated BH Curves of $20 \mathrm{~mm}$ Core $\left(B_{\max }=1.0 \mathrm{~T}, \mathrm{H}_{0}=1.97 \times 10^{-1}\right)$

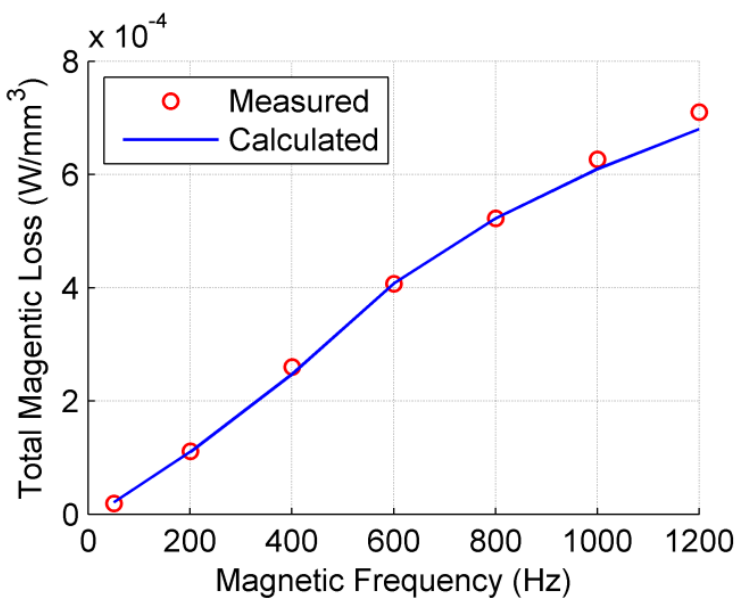

Fig. 12 Dynamic Magnetic Power Loss for $20 \mathrm{~mm}$ Core $\left(B_{\text {max }}=1.0 T, H_{0}=1.97 \times 10^{-1}\right)$

It can be seen from the figures that the $\mathrm{BH}$ curves will gradually become more elliptical than the standard $\mathrm{BH}$ curves with increasing frequency. Although the $\mathrm{BH}$ curve fitting becomes worse in the elliptical frequency range, the energy fitting is still accurate up to $1200 \mathrm{~Hz}$.

Our simulation software can solve the standard BH curves for any given frequency. However, it cannot solve the highly elliptical BH curves higher than $1200 \mathrm{~Hz}$ in this case, due to the limitation of the solving engine. We anticipate that the accuracy of the dynamic Jiles-Atherton model would still be good for higher frequencies if a better solving engine could be utilized.

The accuracy of each calculated magnetic power loss has been calculated based on:

$$
\text { Accuracy, } \%=\left[1-\frac{\left|\boldsymbol{P}_{v, \text { measured }}-\boldsymbol{P}_{v, \text { calculated }}\right|}{\boldsymbol{P}_{v, \text { measured }}}\right] \times 100 \%
$$

The results are summarized in Table 2. It can be seen that the accuracies of most of the calculations are $95 \%$ or above, except for the $50 \mathrm{~Hz}$ ones. The reduced accuracy at lower frequency is possibly caused by the approximation of using the $5 \mathrm{~Hz} \mathrm{BH}$ curve for the static parameter identification. Quasi-static BH curves would therefore be preferable for parameter identification for accurate low frequency modeling. 
Table 2

\% ACCURACY OF THE CALCULATED RESULTS FOR 20 MM LAMINATION STACK

\begin{tabular}{ccccc}
\hline & $1.0 \mathrm{~T}$ & $1.2 \mathrm{~T}$ & $1.4 \mathrm{~T}$ & $1.6 \mathrm{~T}$ \\
\hline $50 \mathrm{~Hz}$ & 88.1 & 88.0 & 86.6 & 95.4 \\
$200 \mathrm{~Hz}$ & 99.1 & 99.9 & 100 & 95.7 \\
$400 \mathrm{~Hz}$ & 95.1 & 99.3 & 98.9 & 94.1 \\
$600 \mathrm{~Hz}$ & 95.9 & 98.7 & 97.9 & 97.0 \\
$800 \mathrm{~Hz}$ & 99.9 & 98.9 & 97.0 & 99.2 \\
$1000 \mathrm{~Hz}$ & 97.3 & 99.9 & 96.2 & 99.1 \\
$1200 \mathrm{~Hz}$ & 95.8 & 96.0 & 95.6 & 98.3 \\
$1400 \mathrm{~Hz}$ & - & - & - & 97.6 \\
$1600 \mathrm{~Hz}$ & - & - & - & 96.9 \\
$1800 \mathrm{~Hz}$ & - & - & - & 98.8 \\
\hline \hline
\end{tabular}

However, over our frequency range of interest, which is $400 \mathrm{~Hz}$ to $2000 \mathrm{~Hz}$, the influence is negligible because the increased dynamic losses dominate over the small differences between the quasi-static and $5 \mathrm{~Hz}$ situations.

\section{B. Core Losses of Rotating PMSM and Agreement with Modelling Above}

In terms of the experimental measurements of the PMSM losses, we used the back-to-back motor test system illustrated in Fig. 13, under open load conditions. The rotational speed and torque of the sample machine are measured to determine the power absorbed. For the BLDC motors under test, which have 7 rotor pole pairs, the maximum magnetic field frequency measured of $1900 \mathrm{~Hz}$ corresponds to a rotational speed of just over 16,200 rpm.

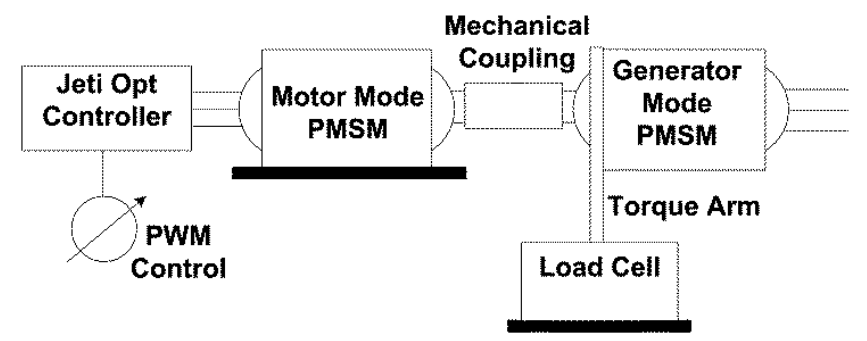

Fig. 13 Test System of PMSM

To measure the magnetic losses of the electrical machine, we firstly measured the total loss of the PMSM comprising the magnetic loss and mechanical loss, then we replace the laminated stator core with a 3-d printed plastic core of the same shape to measure the mechanical loss. Finally, the purely magnetic loss of the laminations is determined by subtracting the mechanical loss from the total loss.

The measured and calculated results for the PMSM with core stacks of $10 \mathrm{~mm}$ and $20 \mathrm{~mm}$ can be seen in Fig. 14 and Fig. 15.

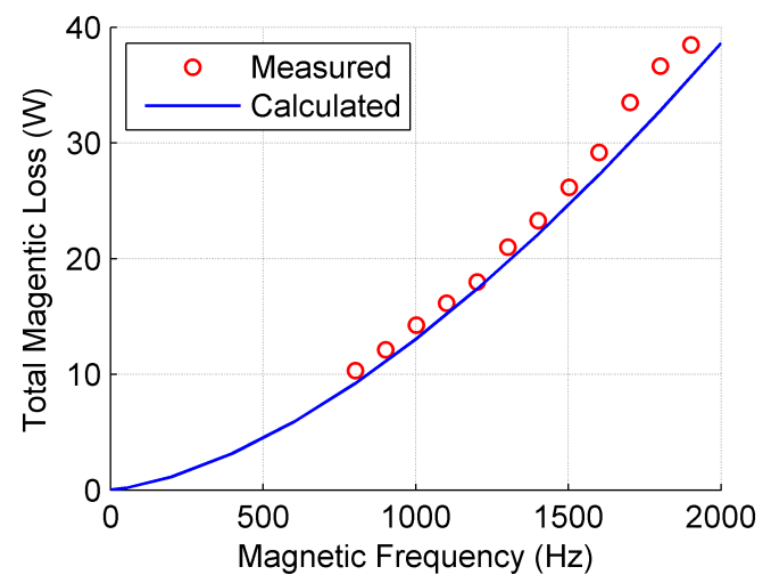

Fig. 14 Magnetic Loss of PMSM with $10 \mathrm{~mm}$ Core

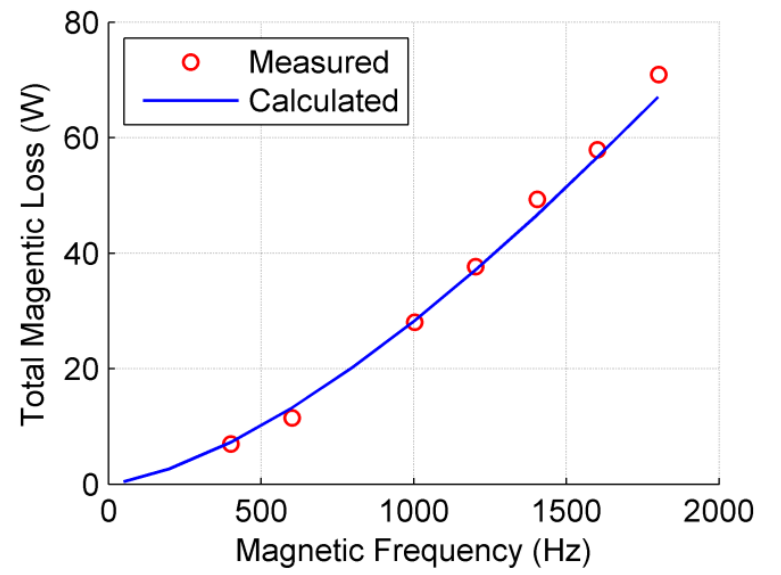

Fig. 15 Magnetic Loss of PMSM with $20 \mathrm{~mm}$ Core

The measured and calculated magnetic losses can be seen to match closely over the frequency range considered. For the example PMSM machines (BLDC motors with a frame size of $50 \mathrm{~mm}$ and lamination stacks of $10 \mathrm{~mm}$ and $20 \mathrm{~mm}$ ) the magnetic losses $\sim 40-80 \mathrm{~W}$ represent about $5 \%$ of the maximum motor rating of $800-1600 \mathrm{~W}$.

\section{SUMmARY \& CONCLUSIONS}

In this paper, a modified dynamic Jiles-Atherton model has been presented to calculate the $\mathrm{BH}$ curves and magnetic power losses in laminated steel. Only two measured $\mathrm{BH}$ curves are required for any given $B_{\max }$ to simulate the dynamic properties of the selected laminated steel over a wide frequency range. The calculated $\mathrm{BH}$ curves, in combination with the flux density distribution in the stator, were used to derive the magnetic power losses in an example machine, a BLDC motor, via the following steps:

1. FEM was used to find the distribution of the magnetic flux density $\mathrm{B}_{\max }$ in the motor stator

2. The functional volume of the laminated steel was then determined from the FEM flux density distribution

3. The BH curve of the lamination stack was measured at low frequency for the stipulated $\mathrm{B}_{\max }$ 
4. The static Jiles-Atherton parameters from the measured $\mathrm{BH}$ curve were then determined via the PSO method

5. A high frequency dynamic $\mathrm{BH}$ curve of the same lamination stack was measured for the same $\mathrm{B}_{\max }$

6. The $H_{0}$ value for the dynamic Jiles-Atherton model was also determined via the PSO method

7. Dynamic BH curves could then be generated for any reasonable frequency via the dynamic Jiles-Atherton Model

8. The total magnetic power loss of the motor was subsequently calculated for a range of speeds, using equation (16).

The calculated magnetic losses have shown around 95\% accuracy over a wide range of frequencies between $5 \mathrm{~Hz}$ and $2,000 \mathrm{~Hz}$ with a magnetic flux density from $1.0 \mathrm{~T}$ to $1.6 \mathrm{~T}$, when compared with experimental measured values.

Although this new approach neglects a number of factors including slot harmonics and power losses at low flux density, it can still yield accurate results over a wide speed range and is computationally fast. Therefore, it is suitable for machine design optimization in which the designer is altering the machine parameters continuously and then running simulations to see if the efficiency has improved or worsened.

Furthermore, this dynamic Jiles-Atherton model has the potential to be extended to calculate the $\mathrm{BH}$ curves for rotational magnetic fields, based on vector generalization [33], [34], or implemented in a time-stepped finite element analysis such as the inverse Jiles-Atherton model [35].

\section{APPENDIX}

\section{A. PSO Method for Parameter Identification in the Static Jiles-Atherton Model}

PSO is an evolutionary computation technique developed by Kennedy and Eberhart [21]. This concept originated as a simulation of a simplified social system, but has since been found effective for engineering optimization [21], [36].

The basic principle of the PSO is similar to the foodseeking process of a swarm. Assuming a swarm consists of $\mathrm{N}$ individuals looking for a "cornfield" in an S dimensional space, and each individual can "remember" its personal best position "pbest[ ]" and the globally best position "gbest[ ]". Kennedy and Eberhart [21] pointed out that the swarm will eventually land on the cornfield if the velocity of the swarm is set as

$$
\begin{aligned}
\mathbf{v}[]=\mathbf{w} * \mathbf{v}[ & ]+\mathbf{c}_{\mathbf{p}} * \operatorname{rand}() \\
& *(\mathbf{p b e s t}[]-\operatorname{present}[])+\mathbf{c}_{\mathbf{g}} \\
& * \operatorname{rand}() \\
& *(\operatorname{gbest}[]-\operatorname{present}[])
\end{aligned}
$$

Where $v[]$ is the velocity matrix of the swarm, present [ ] is the present position matrix of the swarm, rand( ) is a random number between 0 and $1 . \mathrm{w}$ is inertia weight proposed by Shi and Eberhart [37], and they recommended that a w decreasing linearly from 0.9 to 0.4 will work properly for most applications [36], [38]. $c_{p}$ and $c_{g}$ are acceleration constants which represent the weighting of the stochastic acceleration terms that pull each particle toward pbest[ ] and gbest[ ]. By analyzing the simulation results, Kennedy and Eberhart [21] concluded that approximately equal values of the $c_{p}$ and $\mathrm{c}_{\mathrm{g}}$ seem to result in the most effective search of the problem domain, and a default value of $c_{p}=c_{g}=2$ is preferred by them because it works very well for all the types of simulations they have done [36].

The population size of the particles is problem-dependent, and the recommended value was initially 15 to 30 [21], and then increased to 20 to 50 [36]. Actually, the population size of 30 is very commonly used in practical simulations [38], which has also been used in this research project.

A constraint function is used to evaluate if the current position is better or worse, and the "pbest[ ]" "gbest[ ]" will be replaced by the current ones if they are better.

$$
\text { fitness }=\mathbf{f}_{\text {constraint }}(\mathbf{x}[\quad])
$$

Where $x[]=x[]+v[]$ is the current position of the swarm.

When using the PSO method in an S dimensional space, a reasonable searching range should be pre-determined in each dimension in order to speed up the simulation and prevent over searching. In terms of the Jiles-Atherton model parameters, the value of $\mathrm{M}_{\mathrm{s}} \cong \mathrm{B}_{\mathrm{s}} / \mu_{0}=2.05 \mathrm{~T} /\left(4 \pi \times 10^{-7} \mathrm{H}\right.$. $\left.\mathrm{m}^{-1}\right) \cong 1.631 \times 10^{6} \mathrm{~A} \cdot \mathrm{m}^{-1}$ has been given by the manufacturer [39]. The range of $c$ has been given by Jiles and Atherton [4] as $(0,1)$. According to Lederer's research, the parameter $\mathrm{k}$ is the same as the coercive field strength $\mathrm{H}_{C}[15]$. However, referring back to Jiles' work [14], we find the value of $\mathrm{k}$ is quite close to the value of $\mathrm{H}_{C}$ within a difference of around $10 \%$. Therefore, we set the searching range of $\mathrm{k}$ to be $\left(80 \% \mathrm{H}_{C}, 120 \% \mathrm{H}_{C}\right)$ as a reasonable range. In terms of $\alpha$ and a, we use a very large range to cover almost all ferromagnetic materials. The searching ranges of the 5 parameters are listed in Table 3.

Table 3

SEARCHING RANGE OF JILES-ATHERTON PARAMETERS

\begin{tabular}{cc}
\hline \hline Parameter & Range \\
\hline$\alpha$ & {$\left[10^{-7}, 10^{-2}\right]$} \\
$\mathrm{a}$ & {$\left[10^{-1}, 10^{4}\right]$} \\
$\mathrm{c}$ & {$\left[10^{-4}, 0.9999\right]$} \\
$\mathrm{k}$ & {$\left[0.8 \times \mathrm{H}_{C}, \quad 1.2 \times \mathrm{H}_{C}\right]$} \\
$\mathrm{M}_{S}$ & $1.631 \times 10^{6}$ \\
\hline \hline
\end{tabular}

The $\mathrm{BH}$ curve fitting constraint function is set as

fitness $=\frac{1}{N} \sqrt{\sum_{i=1}^{N}\left(\frac{B_{\text {measured }}(i)-B_{\text {calculated }}(i)}{\max \left(B_{\text {measured }}\right)}\right)^{2}}$

This PSO model can then be used with the static Jiles- 
Atherton Model to determine the 4 unknown parameters of the Jiles-Atherton Model.

\section{B. PSO Method for Parameter Identification in the Dynamic Jiles-Atherton Model}

We use the same static parameter array of $\alpha, \mathrm{a}, \mathrm{c}, \mathrm{k} \& \mathrm{M}_{s}$ for the dynamic Jiles-Atherton model at each magnetic flux density step to keep the model consistent. Therefore, only one additional parameter $\mathrm{H}_{0}$ will be determined based on a high frequency dynamic $\mathrm{BH}$ curve.

Since we use a $5 \mathrm{~Hz}$ BH curve for the static parameter identification, which will reduce the accuracy at lower frequency, we should use a high frequency BH curve where the increased dynamic losses dominate over the small differences between the quasi-static and $5 \mathrm{~Hz}$ situations; in order to avoid an inherited error from the static parameter identification process. On the other hand, our Matlab/ Simulink system is not able to solve extreme $\mathrm{H}_{0}$ values at very high frequency. Therefore, a reasonable frequency range should be selected for the $\mathrm{H}_{0}$ identification process, and $150 \mathrm{~Hz} \leq f_{m} \leq 250 \mathrm{~Hz}$ has been found to be a suitable range in our case.

According to the original publication concerning the anomalous loss [9], the value of $\mathrm{H}_{0}$ for the measured laminated steel is normally around $0.06 \mathrm{~A} / \mathrm{m}$ to $0.15 \mathrm{~A} / \mathrm{m}$. Therefore, we choose the searching range as $\left[10^{-6}, 10\right]$ in the PSO method.

Since the anomalous loss was originally proposed to calculate the magnetic power loss rather than the magnetic $\mathrm{BH}$ curve [7], [8], [9], it is better to use an energy fitting constraint function rather than a $\mathrm{BH}$ curve fitting constraint function for the $\mathrm{H}_{0}$ determination. The energy fitting constraint function is set as

$$
\text { fitness }=\frac{\left|\mathbf{P}_{v, \text { measured }}-\mathbf{P}_{v, \text { calculated }}\right|}{\mathbf{P}_{v, \text { measured }}}
$$

Where $\mathrm{P}_{v \text {,measured }}$ and $\mathrm{P}_{v \text {,calculated }}$ can be calculated according to (17).

This PSO model can then be used with the dynamic JilesAtherton Model to determine the unknown parameter $\mathrm{H}_{0}$.

\section{REFERENCES}

[1] Chas. P. Steinmetz, "On the law of hysteresis," Am. Inst. Electr Eng. Trans., vol. IX, no. 1, pp. 1-64, Jan. 1892.

[2] P. Pillay, "An improved formula for lamination core loss calculations in machines operating with high frequency and high flux density excitation," in Conference Record of the 2002 IEEE Industry Applications Conference. 37th IAS Annual Meeting (Cat. No.02CH37344), 2002, pp. 759-766.

[3] D. M. Ionel, M. Popescu, S. J. Dellinger, T. J. E. Miller, R. J. Heideman, and M. I. McGilp, "On the variation with flux and frequency of the core loss coefficients in electrical machines," IEEE Trans. Ind. Appl., vol. 42, no. 3, pp. 658-667, May 2006.

[4] D. C. Jiles and D. L. Atherton, "Theory of ferromagnetic hysteresis," J. Magn. Magn. Mater., vol. 61, no. 1-2, pp. 48-60, Sep. 1986.

[5] S. Chikazumi, Physics of Magnetism. Tokyo, Japan: John Wiley \& Sons. Inc., 1964

[6] C. D. Graham, "Physical origin of losses in conducting ferromagnetic materials (invited)a)," J. Appl. Phys., vol. 53, no. 11, p. 8276, Nov. 1982.
[7] G. Bertotti, "Space-time correlation properties of the magnetization process and eddy current losses: Applications. I. Fine wall spacing," J. Appl. Phys., vol. 55, no. 12, p. 4339, Jun. 1984.

[8] G. Bertotti, "Physical interpretation of eddy current losses in ferromagnetic materials. I. Theoretical considerations," J. Appl. Phys., vol. 57, no. 6, p. 2110, Mar. 1985.

[9] G. Bertotti, "General properties of power losses in soft ferromagnetic materials," IEEE Trans. Magn., vol. 24, no. 1, pp. 621-630, 1988

[10] D. Lin, P. Zhou, W. N. Fu, Z. Badics, and Z. J. Cendes, "A Dynamic Core Loss Model for Soft Ferromagnetic and Power Ferrite Materials in Transient Finite Element Analysis," IEEE Trans. Magn., vol. 40, no. 2, pp. 1318-1321, Mar. 2004.

[11] D. M. Ionel, M. Popescu, M. I. McGilp, T. J. E. Miller, S. J. Dellinger, and R. J. Heideman, "Computation of core losses in electrical machines using improved models for laminated steel," IEEE Trans. Ind. Appl., vol. 43, no. 6, pp. 1554-1564, 2007.

[12] F. Fiorillo and a. Novikov, "An improved approach to power losses in magnetic laminations under nonsinusoidal induction waveform," IEEE Trans. Magn., vol. 26, no. 5, pp. 2904-2910, 1990.

[13] D. C. Jiles, "Frequency dependence of hysteresis curves in conducting magnetic materials," J. Appl. Phys., vol. 76, no. 10, p. 5849, Nov. 1994

[14] D. C. Jiles, J. B. Thoelke, and M. K. Devine, "Numerical determination of hysteresis parameters for the modeling of magnetic properties using the theory of ferromagnetic hysteresis," IEEE Trans. Magn., vol. 28, no. 1, pp. 27-35, 1992.

[15] D. Lederer, H. Igarashit, A. Kost, T. Honmat, and A. Formulation, "On the parameter identification and application of the JilesAtherton hysteresis model for numerical modelling of measured characteristics," IEEE Trans. Magn., vol. 35, no. 3, pp. 1211-1214, 1999.

[16] P. R. Wilson, J. N. Ross, A. D. Brown, and S. Member, "Optimizing the Jiles-Atherton model of hysteresis by a genetic algorithm," IEEE Trans. Magn., vol. 37, no. 2, pp. 989-993, 2001

[17] M. Toman, G. Stumberger, and D. Dolinar, "Parameter identification of the Jiles-Atherton hysteresis model using differential evolution," IEEE Trans. Magn., vol. 44, no. 6, pp. 1098-1101, Jun. 2008.

[18] K. Chwastek and J. Szczygłowski, "An alternative method to estimate the parameters of Jiles-Atherton model," J. Magn. Magn. Mater., vol. 314, no. 1, pp. 47-51, Jul. 2007.

[19] E. Del Moral Hernandez, C. S. Muranaka, and J. R. Cardoso, "Identification of the Jiles-Atherton model parameters using random and deterministic searches," Phys. B Condens. Matter, vol. 275, no. 1-3, pp. 212-215, Jan. 2000.

[20] R. Marion, R. Scorretti, N. Siauve, M.-A. Raulet, and L. Krahenbiihl, "Identification of Jiles-Atherton model parameters using particle swarm optimization," IEEE Trans. Magn., vol. 44, no. 6, pp. 894-897, Jun. 2008.

[21] J. Kennedy and R. Eberhart, "Particle swarm optimization," in 1995 IEEE International Conference on Neural Networks (ICNN 95), 1995, pp. 1942-1948.

[22] R. Eberhart and J. Kennedy, "A new optimizer using particle swarm theory," MHS'95. Proc. Sixth Int. Symp. Micro Mach. Hum. Sci., pp. 39-43, 1995.

[23] D. C. Jiles, "A self consistent generalized model for the calculation of minor loop excursions in the theory of hysteresis," IEEE Trans. Magn., vol. 28, no. 5, pp. 2602-2604, 1992.

[24] K. H. Carpenter, "A differential equation approach to minor loops in the Jiles-Atherton hysteresis model," IEEE Trans. Magn., vol. 27, no. 6 , pp. 4404-4406, 1991.

[25] A. Benabou, J. V. Leite, S. Clénet, C. Simão, and N. Sadowski, "Minor loops modelling with a modified Jiles-Atherton model and comparison with the Preisach model," J. Magn. Magn. Mater., vol. 320, no. 20, pp. e1034-e1038, Oct. 2008.

[26] Youguang Guo, Jian Guo Zhu, Jinjiang Zhong, Haiyan Lu, and Jian Xun Jin, "Measurement and modeling of rotational core losses of soft magnetic materials used in electrical machines: a review," IEEE Trans. Magn., vol. 44, no. 2, pp. 279-291, Feb. 2008.

[27] J. Zhu and V. S. Ramsden, "Improved formulations for rotational core losses in rotating electrical machines," IEEE Trans. Magn., vol. 34, no. 4, pp. 2234-2242, Jul. 1998. 
[28] N. Schmidt and H. Guldner, "A simple method to determine dynamic hysteresis loops of soft magnetic materials," IEEE Trans. Magn., vol. 32, no. 2, pp. 489-496, Mar. 1996.

[29] A. Salvini and F. R. Fulginei, "Genetic algorithms and neural networks generalizing the Jiles-Atherton model of static hysteresis for dynamic loops," IEEE Trans. Magn., vol. 38, no. 2, pp. 873-876, Mar. 2002

[30] Z. Cheng, N. Takahashi, B. Forghani, Y. Du, Y. Fan, L. Liu, Z. Zhao, and H. Wang, "Effect of Variation of B-H Properties on Loss and Flux Inside Silicon Steel Lamination," IEEE Trans. Magn., vol. 47, no. 5, pp. 1346-1349, May 2011.

[31] L. Santi, R. L. Sommer, A. Magni, G. Durin, F. Colaiori, and S. Zapperi, "Dynamic hysteresis in finemet thin films," IEEE Trans. Magn., vol. 39, no. 5, pp. 2666-2668, Sep. 2003.

[32] A. Savitzky and M. J. E. Golay, "Smoothing and differentiation of data by simplified least squares procedures.," Anal. Chem., vol. 36, no. 8, pp. 1627-1639, Jul. 1964.

[33] A. J. Bergqvist, "A simple vector generalization of the JilesAtherton model of hysteresis," IEEE Trans. Magn., vol. 32, no. 5, pp. 4213-4215, 1996.

[34] J. V. Leite, N. Sadowski, P. Kuo-peng, N. J. Batistela, J. P. A. Bastos, and A. A. De Espindola, "Inverse Jiles-Atherton vector hysteresis model," IEEE Trans. Magn., vol. 40, no. 4, pp. 17691775, 2004.

[35] N. SADOWSKI, N. J. BATISTELA, J. P. A. BASTOS, and M. LAJOIE-MAZENC, "An inverse Jiles-Atherton model to take into account hysteresis in time-stepping finite-element calculations," IEEE Trans. Magn., vol. 38, no. 2, pp. 797-800.

[36] R. Eberhart and Y. Shi, "Particle swarm optimization: Developments, applications and resources," in Proceedings of The 2001 Congress On Evolutionary Computation, 2001, pp. 81-86.

[37] Y. Shi and R. Eberhart, "A modified particle swarm optimizer," in 1998 IEEE International Conference on Evolutionary Computation Proceedings. IEEE World Congress on Computational Intelligence (Cat. No.98TH8360), 1998, pp. 69-73.

[38] R. C. Eberhart and Y. Shi, "Comparing inertia weights and constriction factors in particle swarm optimization," in Proceedings of the 2000 Congress on Evolutionary Computation. CECOO (Cat. No.00TH8512), 2000, pp. 84-88.

[39] Nonoriented Electrical Steel, AK Steel Co., West Chester, OH , 2007

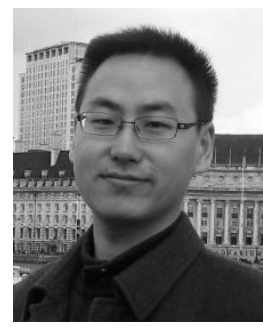

Ruoyang Du (S'14)received the B. Eng. degree in electronic information engineering from Zhejiang University, Zhejiang, China, in 2010 and the M. Eng. degree in electrical engineering from the University of Cambridge, UK, in 2012.

$\mathrm{He}$ is currently pursuing the Ph.D. degree in electrical engineering at the University of Cambridge, UK. His research interests include permanent magnetic synchronous machine design\& modeling, power conditioning circuit design, and combined heat and power system design.

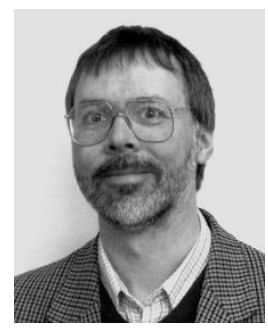

Paul Robertsonreceived the BA degree in Electrical Sciences from the Univeristy of Cambridge, Cambridge, UK in 1984 followed by a $\mathrm{PhD}$ on thin film electronics in 1987 from the same institution.

From 1987 to 1995 he worked at the PA Consulting Group technology center in Melbourn before returning to the faculty in the Engineering Department at the University of Cambridge. He is author of over 50 technical papers and patents. His current research interests include combined heat \& power systems, hybrid electric propulsions systems for aircraft and electromagnetic sensor systems for instrumentation. He has been a Chartered Engineer and Member of the Institution of Engineering and Technology (IET) since 1995.

Dr Robertson was recipient of the SET Awards World Leadership Forum Lecturer of the Year Award in 2005. 